\title{
THE ANSTO ISOTOPE CYCLING SYSTEM
}

\author{
G C Watt ${ }^{1,2} \cdot \mathrm{S}_{\text {Boronkay }}^{3} \cdot$ A M Smith $^{1} \bullet$ M A C Hotchkis ${ }^{1}$
}

ABSTRACT. A number of electronic systems are used on the ANTARES accelerator at ANSTO to implement its fast cycling accelerator mass spectrometry (AMS) capability. The fast cycling system was originally installed and commissioned in 1993 and has recently been updated. This paper describes the more significant of the electronic systems, such as the controller ("sequencer"), the high-voltage power supply ("bouncer"), the fast electrostatic beam chopper, and those used for measurement of the pulsed ion beam current. The sequencer, a programmable 15-bit digital pulse generator, generates the timing and sequencing of the control signals for bouncing voltage selection, beam chopping, Faraday cup current measurement, and rare isotope event measurement. The new sequencer is implemented using a National Instruments FPGA (field programmable gate array) card, programmed using LabVIEW 2010. This device has the benefits of host CPU-independent operation, simple interfacing (PCI), a small footprint, off-the-shelf availability at modest cost, and ease of functionality upgrade. The sequencer provides 15 synchronous digital signals, whose "on" and "off" transitions can be independently specified, in both number and time, with a time resolution of between 0.5 and $128 \mu \mathrm{s}$, and with the total duration between repetitions adjustable between $65.5 \mathrm{~ms}$ and $8.4 \mathrm{~s}$ per cycle. It is hosted by a generic PC because of the low-cost and ubiquity of these. The stand-alone FPGAbased approach ensures that the sequencer determinism is unaffected by processes executing in the host CPU.

\section{INTRODUCTION}

The technique of accelerator mass spectrometry (AMS) is used to measure isotopic ratios, typically of a rare radioactive isotope to a stable isotope. Radiocarbon AMS is an example, where the ratio of the radioactive isotope ${ }^{14} \mathrm{C}\left(\mathrm{t}_{1 / 2}=5730 \mathrm{yr}\right)$ to the stable isotopes $\left({ }^{12} \mathrm{C}\right.$ or $\left.{ }^{13} \mathrm{C}\right)$ is determined. Radioactive and stable isotope(s) must be passed through the accelerator in order to measure their ratio. This has been done in 3 different ways: by "slow cycling", by using a set of recombinator magnets (Litherland and Kilius 1990), or by "fast cycling," also known as "isotope bouncing" (Suter et al. 1983). It is normally the case that the stable beam current exceeds the capability of the accelerator. In this case, a method for reducing the average injected stable isotope currents must also be employed.

The accuracy of the measurement improves with the repetition frequency of isotope cycling, although a trade-off is reached with system dead-time. This improvement is primarily due to better averaging of variations in the ion source output and to a lesser extent on charge stripping and transmission through the accelerator. For this reason, slow cycling, where the injected mass is simply selected by changing injection magnet field, is the poorer choice. These magnets typically have a large inductance and so there are severe limitations on how quickly the magnetic field can be changed. Furthermore, by itself slow cycling does not give any opportunity to attenuate high-current beams.

Recombinator systems, such as that used on the 2MV STAR accelerator at ANSTO, have been developed for measuring ${ }^{14} \mathrm{C}$. These systems first split the beam emerging from the ion source into 3 parallel beams of mass 14,13, and 12. A rotating slotted disc intercepts the abundant mass 12 beam, attenuating it by a factor of 100, before all 3 beams are recombined for simultaneous acceleration. Such systems can only be designed to measure 1 element, and to date all such systems have been designed for carbon.

\footnotetext{
${ }^{1}$ Australian Nuclear Science and Technology Organisation, Lucas Heights 2234, NSW, Australia.

${ }^{2}$ Corresponding author. Email: gwx@ansto.gov.au.

${ }^{3}$ Balaton Technologies, PO Box 368, Rose Bay 2029, NSW, Australia.
}

C 2013 by the Arizona Board of Regents on behalf of the University of Arizona

Proceedings of the 21st International Radiocarbon Conference edited by A J T Jull \& C Hatté

RADIOCARBON, Vol 55, Nr 2-3, 2013, p 308-318 


\section{G C Watt et al.}

Fast cycling systems are much more flexible, allowing a range of elements and their isotopes to be injected sequentially into the accelerator, within the limitations of the available "bouncing" voltage. Furthermore, because the duration that each isotope is injected is adjustable, beam loading of the accelerator can be avoided. The addition of a fast electrostatic beam chopper before the bouncer, as we have on the ANTARES accelerator at ANSTO, provides even more flexibility for beam attenuation. Table 1 lists the isotopes that are commonly measured with the ANTARES system.

Table 1 Isotopes commonly measured at ANTARES using fast isotope cycling. The injection energy is listed along with the species that is selected by the injection magnet, with no voltage applied to the bouncer. The next columns show the species that are "bounced" around the magnet, along with the typical bouncer voltage. For $\mathrm{U}$ and $\mathrm{Pu}$, the injection magnet is set with a 0.5 mass unit offset and a positive voltage applied to the bouncer for all species measured. Also, for $\mathrm{U}$ and $\mathrm{Pu}$, the cycling frequency that can be used is limited by the high-energy bouncing system.

\begin{tabular}{lcllll}
\hline Isotope & $\begin{array}{l}\text { Energy } \\
(\mathrm{keV})\end{array}$ & Magnet set for: & $\begin{array}{l}\text { Cycled isotope, } \\
\text { bouncing voltage } \\
(\mathrm{kV})\end{array}$ & $\begin{array}{l}\text { Additional isotopes, } \\
\text { bouncing voltages } \\
(\mathrm{kV})\end{array}$ & $\begin{array}{l}\text { Cycling } \\
\text { frequency } \\
(\mathrm{Hz})\end{array}$ \\
\hline${ }^{7} \mathrm{Be}$ & 113.7 & ${ }^{9} \mathrm{Be}^{16} \mathrm{O}^{-}$ & ${ }^{7} \mathrm{Be}^{16} \mathrm{O}, 9.8$ & - & 5 \\
${ }^{10} \mathrm{Be}$ & 113.7 & ${ }^{10} \mathrm{Be}^{16} \mathrm{O}^{-}$ & ${ }^{9} \mathrm{Be}^{16} \mathrm{O}, 4.5$ & - & 5 \\
${ }^{14} \mathrm{C}$ & 88.9 & ${ }^{14} \mathrm{C}^{-}$ & ${ }^{13} \mathrm{C}, 6.7$ & ${ }^{12} \mathrm{C}, 14.5$ & 5 \\
${ }^{14} \mathrm{C}$ & 108.5 & ${ }^{14} \mathrm{C}^{-}$ & ${ }^{13} \mathrm{C}, 8.3$ & - & 5 \\
${ }^{26} \mathrm{Al}$ & 88.4 & ${ }^{27} \mathrm{Al}^{-}$ & ${ }^{26} \mathrm{Al}, 3.4$ & - & 5 \\
$\mathrm{U}$ & 100 & mass 252.5 & ${ }^{236} \mathrm{U}^{16} \mathrm{O}, 0.36$ & $\begin{array}{l}{ }^{234} \mathrm{U}^{16} \mathrm{O}, 1.15 \\
{ }^{233} \mathrm{U}^{16} \mathrm{O}, 1.63\end{array}$ \\
$\mathrm{Pu}$ & 100 & mass 258.5 & ${ }^{242} \mathrm{Pu}^{16} \mathrm{O}, 0.32$ & $\begin{array}{l}{ }^{240} \mathrm{Pu}^{16} \mathrm{O}, 1.12 \\
239 \mathrm{Pu}^{16} \mathrm{O}, 1.50\end{array}$ & 0.16 \\
\hline
\end{tabular}

Fast cycling systems rely upon modulating the beam energy before the beam enters the magnet so that specific ion masses in that beam have the right magnetic rigidity to follow the injection trajectory; the other masses are lost from the beam. This is accomplished by using a simple gap lens before the magnet entrance, with the inner electrode connected to the magnet vacuum chamber, which is insulated from the pole pieces. The vacuum chamber is also connected to the inner electrode of a second gap lens at the exit of the magnet; both outer gap lens elements are at ground potential. When the bouncer voltage is applied, the beam energy is changed on passing the first gap lens and returns to its initial value on passing the second gap lens. The gap lenses are placed as close as practical to the object and image planes of the magnet to minimize the focal effect of the gap lenses and because the beam waist is smallest in these positions. The overall capacitance of the bouncing system can be kept quite small, permitting rapid changes in bouncing voltage and so selection of different isotopes.

The electronics of the ANTARES fast cycling system has evolved since it was first described in Smith et al. (1994), although the beam line components have remained essentially unchanged. Figure 1 is a schematic diagram of the ANTARES fast isotope cycling system. The beam chopper vertical deflection plates are mounted just in front of the pre-acceleration tube, so with a modest deflection voltage of $\pm 400 \mathrm{~V}$ the beam is occluded by a vertical slit placed $7 \mathrm{~mm}$ off-axis in the object plane of the magnet. The un-chopped beam passes through the first gap lens just after these slits, around the injection magnet and through the second gap lens, which is just in front of the slits in the image plane. The magnet is provided with an enlarged image chamber with ports for off-axis Faraday cups (not implemented). 


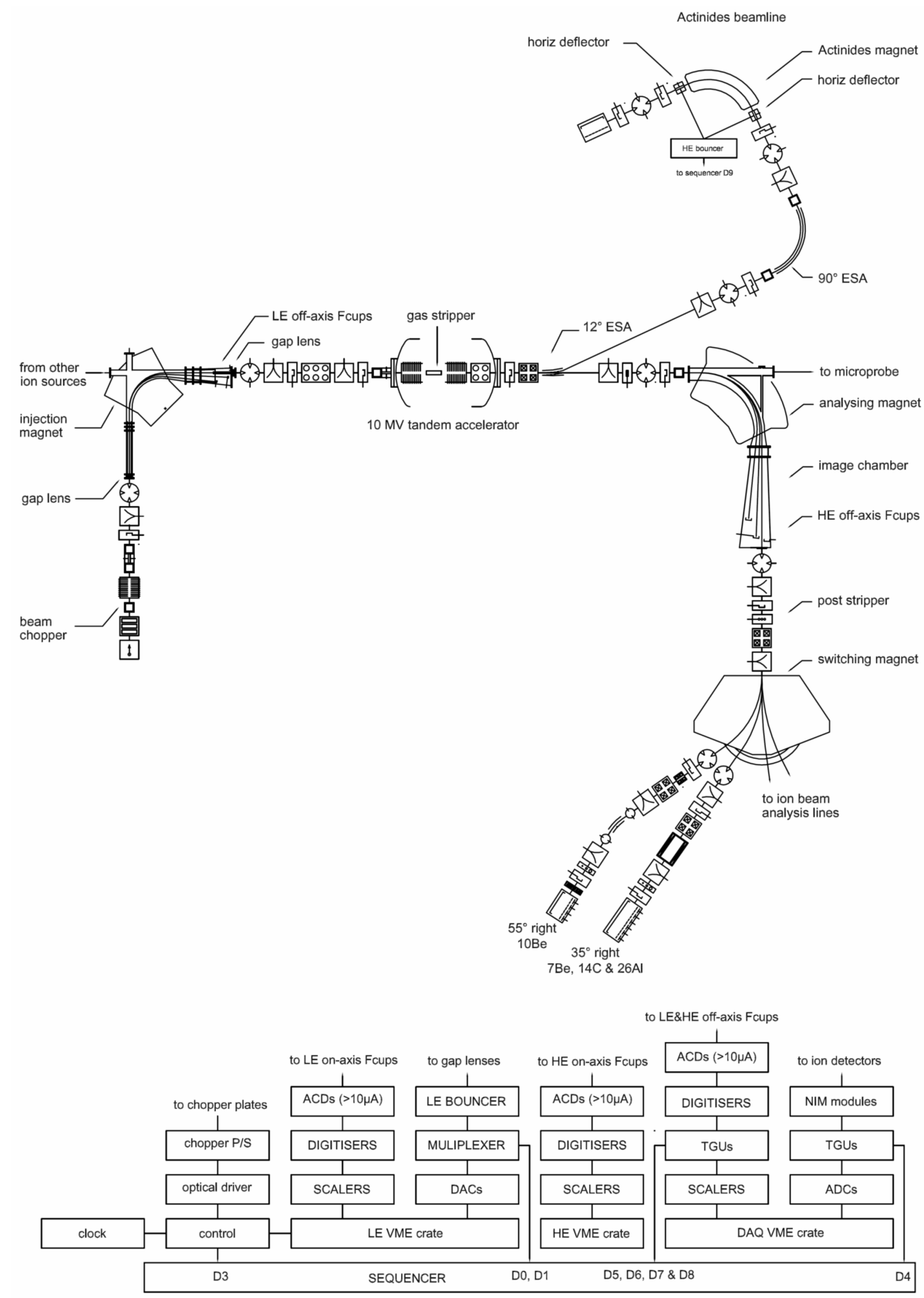

Figure 1 ANTARES fast isotope cycling system, showing the physical layout and relationship between associated electronic systems.

The high-energy analyzing magnet has an enlarged image chamber and is provided with off-axis Faraday cups, adjustable to intercept deflected isotopes, 2 on the low-rigidity side and 1 on the highrigidity side. The stable beam currents are measured using these cups. The rare beam continues through the image plane of the magnet and is guided into an ionization detector. 


\section{GC Watt et al.}

For actinides measurements, a separate high-energy beamline is used, incorporating a high-resolution magnet and a high-energy bouncing system to enable counting of several rare isotopes in 1 ion detector. This is described in the "High-Energy Bouncer" section below.

The present isotope cycling system on ANTARES at ANSTO consists of electronic modules that:

- Deflect the injected beam off-axis ("chopping") with a precisely known duty cycle, during the measurement of beam current;

- Measure off-axis Faraday cup currents, using current digitizers;

- Generate gating signals of accurately known pre-settable durations that synchronize each Faraday cup current measurement and ionization detector events with the on-axis duration of the isotope being measured;

- Generate a fast-rise, accurately settable succession of "bouncing" voltage levels (typically of multi-kilovolt amplitudes) to select the isotope on-axis for measurement;

- Repetitively generates all of the control signals above ("sequencer") deterministically with an accuracy of tens of ppm, $\mu$ s-level time resolution, and ns-level time pulse synchronism, in accordance with measurement parameters entered by the user.

During every counting cycle, provision has been made to measure up to 4 separate isotopes, each with independently settable duration, bouncing voltage, and chopping duty cycle.

\section{ELECTRONICS}

\section{Sequencer}

The sequencer provides 15 programmable output signals, of which 12 perform the dedicated functions listed in the previous section. Three further outputs may be directly programmed by the user, independently of the AMS parameter entry process. All outputs are capable of driving a terminated $50 \Omega$ line to $>4.0 \mathrm{~V}$ in the logic "high" state, and are protected against incoming transients. The rise and fall times are approximately $10 \mathrm{~ns}$.

The original sequencer, as commissioned in 1993, consisted of a $64 \mathrm{k} \times 16$ bit SRAM data memory used as a lookup table. Each of the 16 data bits provided a digital output signal, and the SRAM address was generated by a 16-bit counter clocked at a pre-settable stable frequency, equivalent to the required time resolution. Largely due to component obsolescence, this was replaced late in 2011 by a National Instruments FPGA (Field Programmable Gate Array) card. This card has been programmed using LabVIEW 2010.

For reasons of compatibility, the initial specifications of the FPGA version were chosen to be identical to those of the original system (minimum $0.5 \mu$ s resolution, maximum duration 65,535 clock cycles, 15 output bits, 4 isotopes). However, there is now scope to greatly expand upon this capability. This can be done now by manually editing the programming file used by the sequencer to provide longer counting times, and in the future by reprogramming the FPGA to alter the overall sequencer architecture.

The original SRAM implementation was limited to a maximum cycle duration of (clock period $\times$ $65,536)$ due to the direct mapping of successive SRAM locations to corresponding successive time instants. This not only imposes a fundamental trade-off between duration and resolution, but also results in significant wastage of memory when specific output signal combinations remain constant for a large number of clock cycles, resulting in large blocks of memory containing identical data. 
In contrast, the flexibility of the FPGA architecture overcomes this limitation by implementing a looping structure to determine the duration of each successive output state (a period during which all output signals remain constant). The internal FPGA clock period selected was $0.1 \mu \mathrm{s}$, and the loop counter modulus was chosen to be a 32-bit variable, meaning that the ultimate realizable resolution and minimum and maximum duration capability for any state is now $0.1,0.1$, and $\left(2^{32}-1\right) \times 0.1 \mu \mathrm{s}$ ( $\sim 430$ s), respectively.

Preparation of the FPGA system for fast cycling follows a 2-step process. In the first step, the user enters desired parameters for count durations, chopping duty cycles, ion flight time, bouncer response time, and representative bouncing voltages for each isotope through a Visual Basic 6.0 application, developed in-house. The main screen of the AMS sequencer data entry/review program is shown in Figure 2. In this example, a file commonly used for ${ }^{14} \mathrm{C}$ AMS analysis on ANTARES (Cycle\#29.txt) has been loaded.

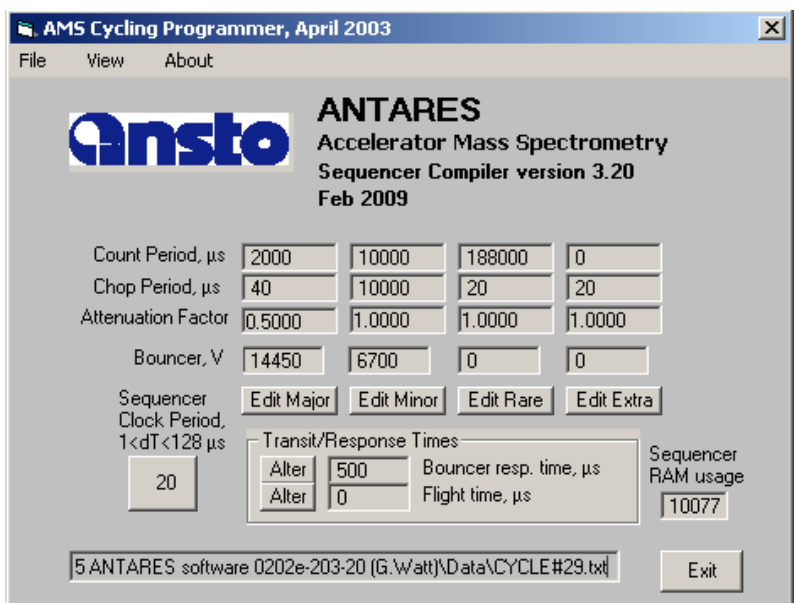

Figure 2 User-screen for data entry and review

This configuration specifies a counting period of $2 \mathrm{~ms}$ for ${ }^{12} \mathrm{C}$ ("Major" isotope; bouncer $=14.45$ $\mathrm{kV}$ ), $10 \mathrm{~ms}$ for ${ }^{13} \mathrm{C}$ ("Minor" isotope; bouncer $=6.70 \mathrm{kV}$ ), and $188 \mathrm{~ms}$ for ${ }^{14} \mathrm{C}$ ("Rare" isotope; bouncer $=0$ ). The actual bouncing voltages are later tuned and set in DACs; these values are for depictive use only. An (excessive) bouncer response time of $500 \mu$ s was allowed between each of these transitions to allow the bouncer voltage to accurately settle to its new value. The "Extra" gating period is not used with carbon measurements.

During the ${ }^{12} \mathrm{C}$ phase, the beam is chopped with a $50 \%$ duty cycle (Attenuation Factor $=0.5000$ ), implemented as a sequence of $20-\mu \mathrm{s}$ on-axis and $20-\mu \mathrm{s}$ off-axis periods (Chop Period $=40 \mu \mathrm{s}$ ). The beam chopper is inactive for ${ }^{13} \mathrm{C}$ and ${ }^{14} \mathrm{C}$. The data entry/review program embodies knowledge of the constraints imposed by the original sequencer architecture and rejects any user values that cannot be supported by the hardware, e.g. use of more than 65,535 memory locations, clock period outside the range 1-128 $\mu \mathrm{s}$, non-integral number of chopping cycles per count period, non-integral number of clock cycles per gate period, and so on. The software will coerce non-allowable values to the nearest allowable values that can be supported by the hardware.

The output of this first step is an ASCII file whose header tabulates the parameters entered by the user, and which then lists pairs of sequencer output state value (bits), and time value (address) at 


\section{GC Watt et al.}

which one or more of the bits next changes value. In the second step, these data, in an appropriate form, are loaded into the FPGA memory, using the file dialog of the LabVIEW user interface created for it. The user interface has a simulated "oscilloscope" function, depicting the time behavior and inter-relationships between all sequencer output signals for the particular counting configuration.

The sequencer uses a National Instruments PCI 7811R PCI-bus Xilinx Virtex 2 FPGA card. The FPGA sequencer was developed for ANSTO during 2011 by Balaton Technologies using the FPGA programming module of LabVIEW 2010. No specialized knowledge of FPGA coding (e.g. VHDL) or of the internal architecture of the FPGA is necessary. Interfacing to the host computer is also simplified because LabVIEW writes the signal states and durations directly into the FPGA memory via the internal PCI backplane interface, rather than via the cumbersome external I/O port interface originally used.

This implementation of the sequencer is externally functionally identical to the one that it replaces, and has a much smaller footprint (it is located within the host PC, instead of in a separate housing). As originally designed, all 15 external outputs of the sequencer are capable of simultaneously driving a $50 \Omega$ external load to $>4.0 \mathrm{~V}(>200 \mathrm{~mA})$. Because the FPGA used has a recommended maximum output current of $5 \mathrm{~mA}$ per channel, a 16-channel discrete-transistor line-driver buffer stage has been provided to increase the available output current. This has the additional advantage of protecting the FPGA. In the event of damaging signals being applied to the output lines (e.g. tank spark transients, unintentional overvoltage, etc.), the inexpensive transistors are the first components affected, and these are easily replaced.

It is envisaged that future developments will provide for measurement of up to 8 isotopes, with possible counting durations between $1 \mu \mathrm{s}$ and $>400$ s per isotope, while maintaining sub- $\mu$ s-level timing resolution and output timing synchronism of $\sim 5 \mathrm{~ns}$. These developments are easily implemented in the new sequencer system, and 10 additional $50 \Omega$ output line drivers have already been provided in anticipation of future upgrading.

Figure 3 shows actual output waveforms from the sequencer, via the $50 \Omega$ line drivers, using a Tektronix TDS 420A DSO, after Cycle\#29.txt has been loaded into the FPGA memory. This shows the voltages present during the measurement of ${ }^{12} \mathrm{C}$, including the pre- and post-500- $\mu$ s delay during the bouncer voltage settling, and the $50 \%$ duty-cycle chopping during the ${ }^{12} \mathrm{C}$ ion current measurement.
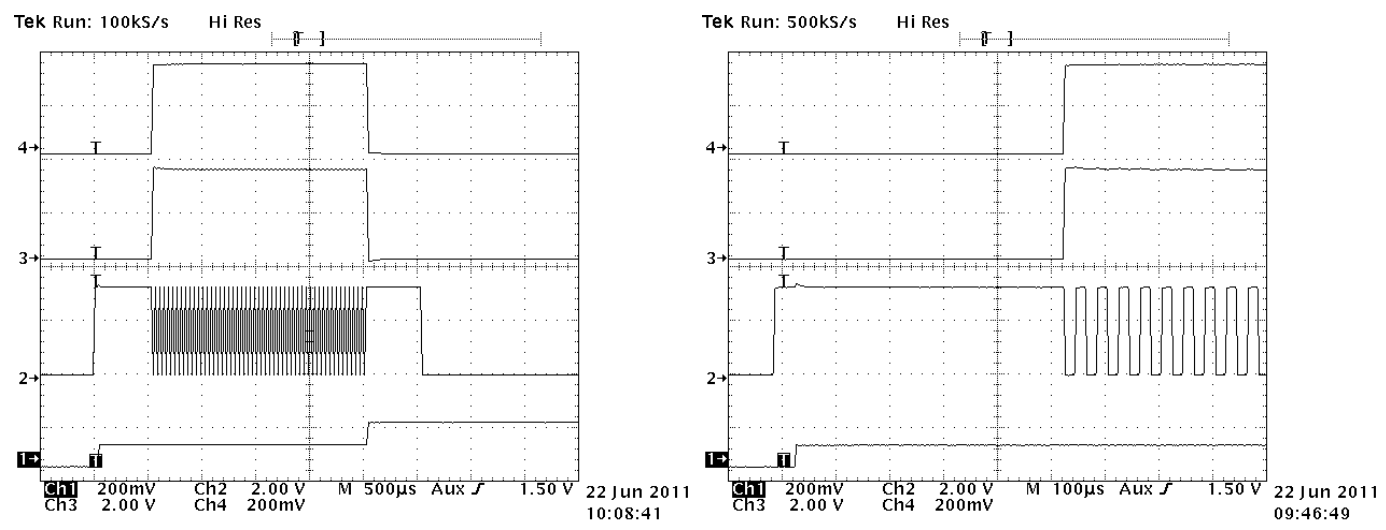

Figure 3 Actual output waveforms from the sequencer showing a ${ }^{12} \mathrm{C}$ counting period, firstly showing the entire duration $(2 \mathrm{~ms})$ in relation to the bouncing voltage transitions, then in $\times 5$ finer detail, showing the chopping at $50 \%$ duty cycle. 


\section{Beam Chopper}

The beam chopper operates on the beam before it enters the pre-acceleration tube on the $846 \mathrm{~B}$ ion source injection module (Figure 1). The beam energy is typically $\sim 30-40 \mathrm{keV}$ at this point so only a modest voltage is required to deflect it off-axis. Two horizontal beam chopper plates are driven in anti-phase to deflect the beam vertically, sweeping it across a slit in the object plane of the injection magnet. A distance of $7 \mathrm{~mm}$ between this slit and the axis is adequate to ensure the beam is completely occluded for a deflection voltage of $\pm 400 \mathrm{~V}$. Zero differential voltage allows the beam to pass undeflected.

The ANTARES computer control system (using the EPICS toolkit running on Linux PCs) allows the selection of 1 of 4 states, presented to the chopper system by 2 address lines. These states are "beam on-axis" (zero chopper voltage), "beam off-axis" (chopper voltages continuously applied), "setup mode," or "sequencer driving chopper." For the "setup mode," a unit was designed with clock periods selectable as $1 \mu \mathrm{s}, 10 \mu \mathrm{s}, 100 \mu \mathrm{s}, 1 \mathrm{~ms}$, or $10 \mathrm{~ms}$, and a duty cycle selectable as $10 \%$ or $1 \%$ to provide fixed ratio attenuation of the beam; this functionality is very useful for tuning up the accelerator under actual measuring conditions, i.e. with the ion source output maximized for high counting rates of the rare isotope and therefore large currents from the stable isotopes. The fourth state, "sequencer driving chopper," accepts the dedicated sequencer output bit to drive the chopping system; this is fully flexible, as described above in the "Sequencer" section.

A block diagram of the chopper system is shown in Figure 4a. In the circuit, switches SW1, 2, 3, and 4 are $1000 \mathrm{~V} \mathrm{~N}$-channel MOSFETs. The circuit operates on the ion source platform at pre-acceleration potential, controlled through fiberoptic links. "Earth" is relative to pre-acceleration potential. Figure $4 \mathrm{~b}$ shows details of one of the chopper stages (some details have been omitted for clarity).

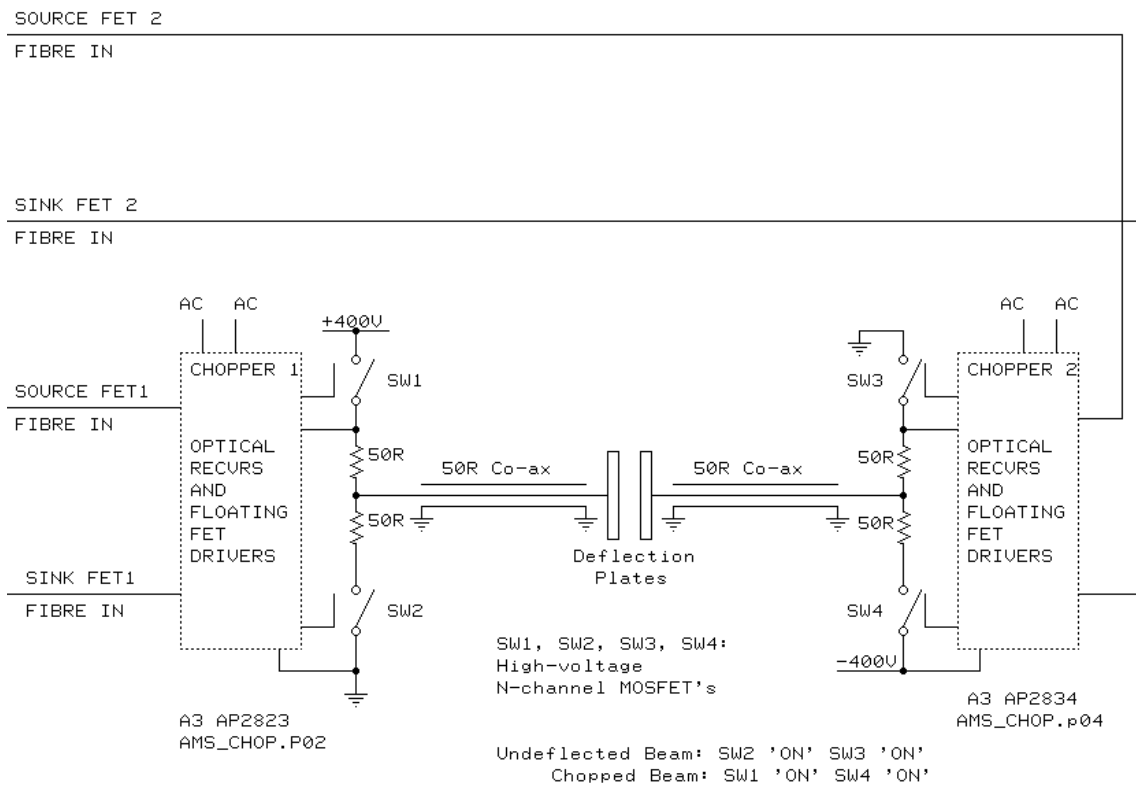

Figure 4(a) Outline circuit of the beam-chopping electronics

The chopping circuit consists of a push-pull pair of BUK456-1000B power MOSFETs operating from low-current $+400 \mathrm{~V}$ and $-400 \mathrm{~V}$ voltage-multiplying DC power supplies. The " $+400 \mathrm{~V}$ " DC 


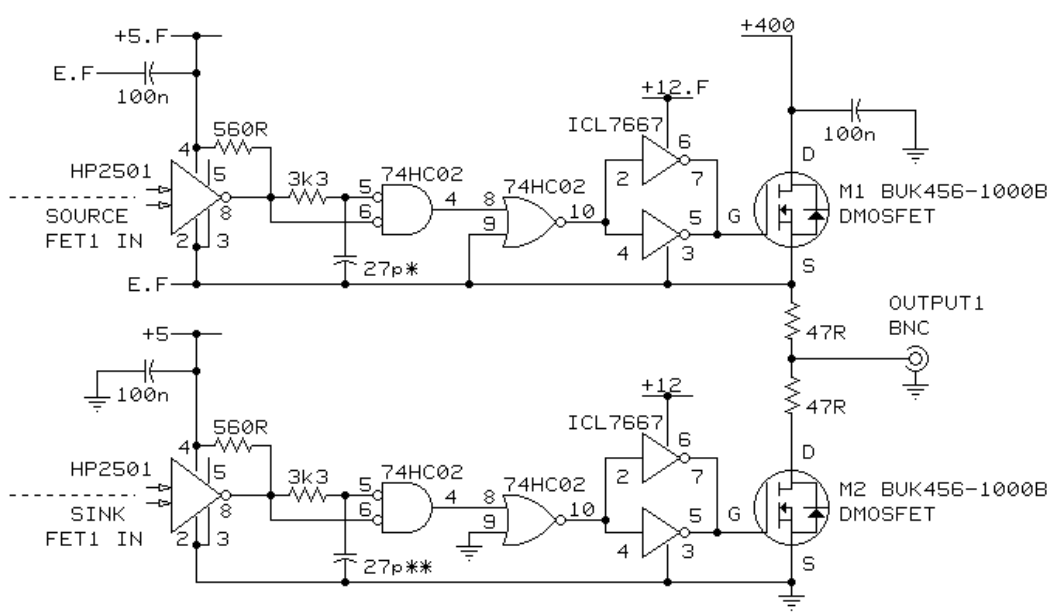

Figure 4(b) Circuit for one of the 2 totem-pole MOSFET choppers

supply is relative to the pre-acceleration potential. The +5 .F and +12 .F supplies are referenced to the source potential of MOSFET M1. The value of the capacitor marked " $27 \mathrm{p} * *$ " was selected to provide approximately $50 \mathrm{~ns}$ of commutation delay between one MOSFET switching off and the other being switched on, to prevent parasitic oscillation. The second chopping circuit is identical except that it operates with supply voltages of zero and $-400 \mathrm{~V} \mathrm{dc}$, relative to pre-acceleration potential. The only electrical loading on the chopper system is the transient current required to charge and discharge the cable capacitance and electrode capacitances. These are of the order of tens of $\mathrm{pF}$. The measured rise and fall times were $10 \mathrm{~ns}$ with $25 \mathrm{pF}$ loading, and a delay time of $220 \mathrm{~ns}$.

As these supplies operate at pre-acceleration potential (up to $150 \mathrm{kV}$ above ground), a means of transmitting the drive pulses from ground potential was required. One-mm diameter Hewlett-Packard HFBR plastic fiberoptic cable and HP 1501/HP 2502 transmitters/receivers were used, following experience with other high-voltage isolation applications at ANSTO. This system has the advantages that fiber termination requires no specialized expertise or equipment, optical loss is modest, meaning that links of several meters are feasible, and the fiberoptic cable has adequate withstand capability for the voltages used.

\section{Current Measurements}

Faraday cup currents are measured by Red Nun RN8111 bipolar current digitizers (actually described by Red Nun as "charge pumps" because of the way they work). These units produce 1 TTL output pulse for each $10 \mathrm{pC}$ of input charge; input currents up to a maximum of $10 \mu \mathrm{A}$ can be measured, corresponding to a $1 \mathrm{MHz}$ output pulse stream. The nonlinearity is better than $0.2 \%$ and the input leakage current is approximately $5 \mathrm{pA}$. Separate discrete logic LSTTL gating circuits are used to ensure that each isotope current is only integrated during its counting period, as determined by the sequencer output gates. The gated pulse streams from the Red Nuns are then accumulated by scalers in the VME-based ANTARES data acquisition system.

The isotope cycling system is operated in such a way as to keep the average injected negative-ion current into the accelerator less than about $1 \mu \mathrm{A}$. To cater for a maximum instantaneous high-energy positive ion beam current of up to $200 \mu \mathrm{A}$, attenuation of the ion current by a factor of 20 was necessary before digitization by the RN8111. Attempts to use a passive-resistive current attenuator 
resulted in substantial errors due to mains-related noise, and degraded input drift and linearity of the RN8111.

These problems were overcome by using an active current attenuator, whose input has low impedance, approximating a virtual earth, and whose output has very high impedance, approximating an ideal current source. The low input resistance ensures fast response and complete current collection from the Faraday cup, important for the pulsed ion beams that enter the Faraday cups. The high output resistance shunting the RN8111 input eliminates the sources of error mentioned above. The circuit used is shown in Figure 5.

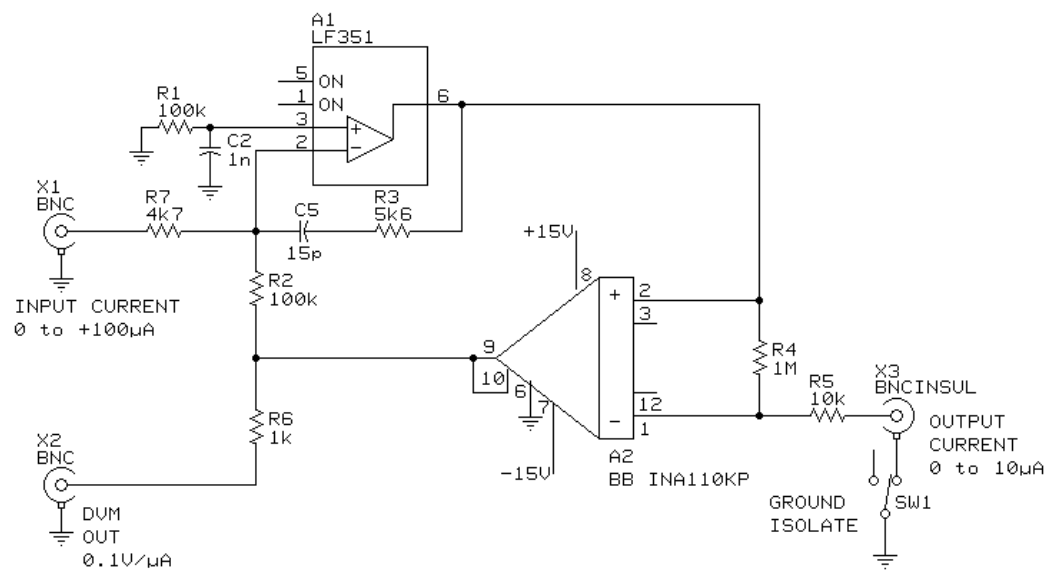

Figure $5 \times 0.1$ active current divider

In this circuit, $A_{1}$ is a high-gain operational amplifier with bias current $<50 \mathrm{pA} ; \mathrm{A}_{2}$ is a differential amplifier with a gain of unity $\pm 0.02 \%$ and $\sim 20 \mathrm{pA}$ bias current. The virtual earth at the input of $\mathrm{A}_{1}$ is maintained by balancing the input current with a current applied by amplifier $\mathrm{A}_{2}$. When the feedback loop is in balance, the voltage across $R_{2}(100 \mathrm{k} \Omega)$ is equal to the drop across the current measurement shunt $R_{4}(1 M \Omega)$, hence the output current is $R_{2} / R_{4} \times$ (input current). For clarity, offsetbalance components are not shown. The measured output resistance was $>1 \mathrm{G} \Omega$. The unit is assembled in a single width NIM module, and is powered by dedicated $\pm 15 \mathrm{~V}$ DC supply rails. It is fitted with a switch that can open the local connection to ground to eliminate any residual ground loop problems if necessary.

\section{Low-Energy Bouncing Amplifier}

In order to minimize dead-time in the isotope bouncing system, it was required that the bouncing voltage source respond to $99.9 \%$ of the target voltage within $0.5 \mathrm{~ms}$ over the $0-15 \mathrm{kV}$ range. Assuming a load capacitance of $2 \mathrm{nF}$, a response time of $100 \mu$ s requires a minimum output slew rate of $150 \mathrm{~V} / \mu \mathrm{s}$, and a required minimum output current of $300 \mathrm{~mA}$.

The repetitive high-voltage bouncing waveform is generated by amplifying a low-voltage analog of the high-voltage waveform. The precision high-voltage operational amplifier (HVA) is a TREK P0842 with an amplification factor of 1500. The HVA has an output current capability of $>300 \mathrm{~mA}$, an output voltage capability of 0 to $+15 \mathrm{kV}$, and a risetime of $\sim 150 \mu$ s into a $2.2 \mathrm{nF}$ load to within $99.9 \%$ of the target voltage. 


\section{GC Watt et al.}

A block diagram of the low-energy bouncing amplifier electronics is shown in Figure 6 . The lowvoltage analog waveform is generated by 4 VME260 12-bit DACs with each of these voltages selected in turn by an analog multiplexer (a high-speed 4-position switch), as determined by the 2bit address code generated by the sequencer. For instrument setup, a scanning routine within the EPICS control system applies each bouncing voltage over a selected range at selectable resolution and rate, allowing the peak or the center of a flat-topped response to be set for the particular DAC.

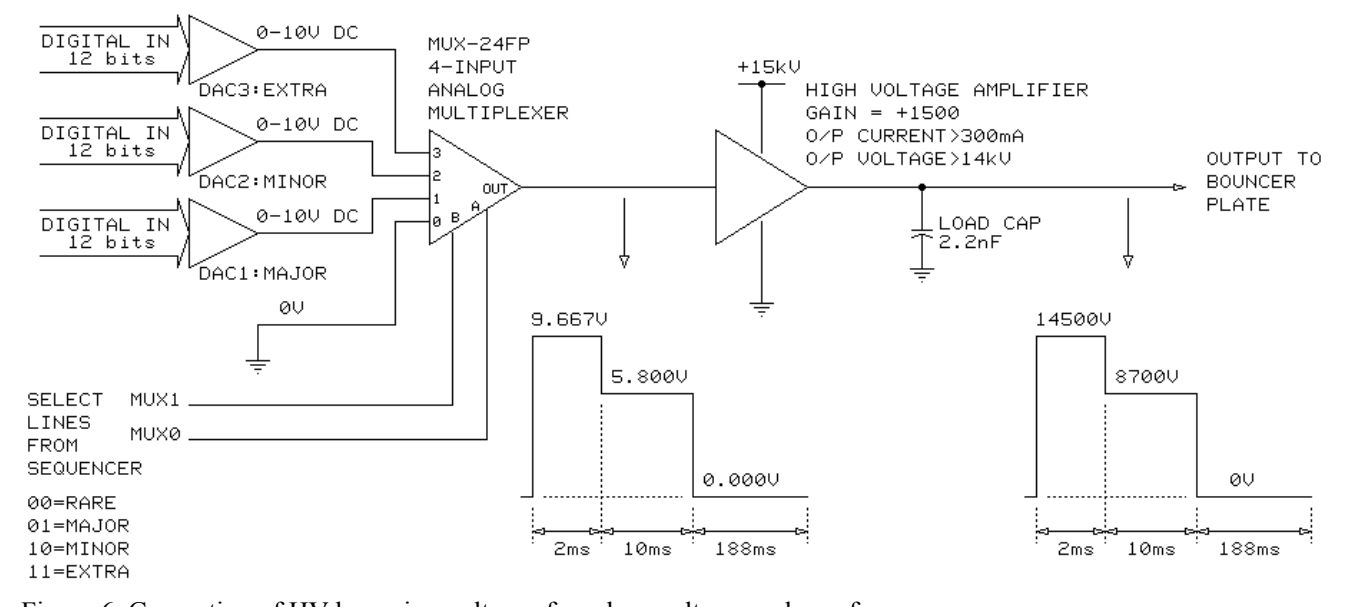

Figure 6 Generation of HV bouncing voltages from low-voltage analog references

Each of the 4 possible multiplexer addresses correspond to the Major, Minor, Rare, or Extra isotope, and selects the applicable 1/1500th of the required high-voltage bouncing voltage for the relevant counting period. The multiplexer uses an Analog Devices MUX-24FP dual-channel 4-input JFET analog multiplexer. Both active and common analog lines are switched, and fed differentially to the differential inputs of the HVA to ensure maximum noise rejection.

\section{High-Energy Bouncer}

For actinides measurements, the same low-energy bouncing system is used, as described above. However, a separate high-energy beamline is used, incorporating high-resolution magnetic and electrostatic analyzers. As it is necessary to measure several rare isotopes in the same ion detector at the focus of the magnet, a high-energy bouncing system has been implemented (Hotchkis et al. 2010). Isotopes of different mass are directed to the detector without changing the magnetic field by applying different voltages to a symmetric pair of horizontal deflector plates before and after the magnet (Zorko et al. 2010). The required voltage pulses to switch between isotopes are supplied by an EPICS-enabled microIOC unit built by Cosylab, Slovenia. The response time of the power supplies in this system is $50 \mathrm{~ms}$. As a result, typical counting times used for actinides isotopes are 1 to $5 \mathrm{sec}-$ onds. Isotope injection is controlled by the low-energy bouncing system. The sequencer provides the necessary gating signals and also a trigger output at the start of each cycle to drive the microIOC unit and keep the LE and HE system in synchrony.

\section{SUMMARY}

The ANTARES isotope cycling system has proven extremely versatile and has allowed the AMS measurement of isotopic ratios of elements from beryllium to the actinides. The electrostatic beam chopper permits operation of the ion source at high output, maximizing the counting rate of the rare 
isotope while maintaining the stable ion beam currents within the limits imposed by the accelerator. The new FPGA-based sequencer has enhanced the versatility of the system and ensures that it can be expanded, serviced, and maintained for the foreseeable future.

As originally envisaged, the system has been designed to permit the measurement of the stable isotopes in off-axis Faraday cups at the injection magnet, while the rare isotope is on-axis for injection into the accelerator. Although the enlarged injection magnet image chamber has been supplied with suitable ports and the electronics and software is ready for these signals, the Faraday cups are yet to be installed. We hope to complete this last stage as it will permit online monitoring of accelerator transmission and will permit high-energy signals to be normalized to ion source output variations

\section{REFERENCES}

Hotchkis MAC, Child DP, Zorko B. 2010. Actinides AMS for nuclear safeguards and related applications. Nuclear Instruments and Methods in Physics Research $B$ 268(7-8):1257-60.

Litherland AE, Kilius LR. 1990. A recombinator for radiocarbon accelerator mass spectrometry. Nuclear Instruments and Methods in Physics Research B 52(34):375-7.

Smith AM, Fink D, Hotchkis MAC, Jacobsen GE, Lawson EM, Shying M, Tuniz C, Watt GC, Fallon J, Ellis PJ. 1994. Equipment and methodology for high precision high throughput ${ }^{14} \mathrm{C}$ AMS analyses at $\mathrm{AN}$ -
TARES. Nuclear Instruments and Methods in Physics Research B 92(1-4):122-8.

Suter M, Balzer R, Bonani G, Nessi M, Stoller C, Woelfli W, Andree M, Beer J, Oeschger H. 1983. Precision measurements of rare radioisotopes with a tandem Van de Graaff accelerator. IEEE Transactions on $\mathrm{Nu}$ clear Science 30(2):1528-31.

Zorko B, Child DP, Hotchkis MAC. 2010. A fast switching electrostatic deflector system for actinide isotopic ratio measurements. Nuclear Instruments and Methods in Physics Research B 268(7-8):827-9. 